\section{Response to comments on: Ocular surface status in patients with hemifacial spasm under long-lasting treatment with botulinum A toxin: A comparative fellow eye study}

\section{Dear Sir,}

First of all, we would like to thank you for the constructive comments ${ }^{[1]}$ on our study on ocular surface status following botulinum A toxin. ${ }^{[2]}$ The authors of the letter pointed out that since the adverse events of botulinum A toxin injection usually occur during the first period of treatment, it could be appropriate to divide our sample according to the number of injections received. Although we have already investigated the correlations between the ocular surface parameters and the duration of treatment, of course a further analysis based also on the number of injections may be of interest. The mean number of injections was 22.3 (range 2-53). The sample size was too small to create 3 different subgroups according to the number of injections, as suggested $(6-12 ; 12-25 ;>25)$. Furthermore, we were also unable to create a control group with patients who received a number of injections comprised between 1 and 3 , because they were only 4 . Therefore, we divided overall patients into 2 groups: Those who received less than 25 injections $(n=14)$ and those who received 25 or more injections $(n=12)$. Tear osmolarity was significantly lower in patients who received less than 25 injections (291.6 \pm 22.7 vs $309.3 \pm 9.9 \mathrm{mOsm} / \mathrm{L}, P=0.017)$. This result is in agreement with the correlation between tear osmolarity and the duration of treatment already found in our study. Conversely, we did not find any significant difference for tear film break-up time, Schirmer test, corneal sensitivity, corneal staining, and OSDI score between the two groups (all $P>0.05$ ).

In conclusion, the relationship between the number of botulinum A toxin injections and the ocular surface changes is not fully clarified by our study and further prospective studies with larger sample size are required to elucidate this issue. The evaluation of other ocular surface parameters not investigated in this paper, such as Jones test, could provide further useful information.

Financial support and sponsorship

Nil.

\section{Conflicts of interest}

There are no conflicts of interest.

\section{Marco Pellegrini, Costantino Schiavi, Leonardo Taroni, Stefano Sebastiani, Federico Bernabei, Matilde Roda, Fabiana Moscardelli, Giuseppe Giannaccare}

Department of Ophthalmology, S. Orsola-Malpighi University Hospital, University of Bologna, Bologna, Italy

Correspondence to: Dr. Marco Pellegrini, Ophthalmology Unit, S. Orsola-Malpighi University Hospital, University of Bologna, Via Palagi 9, Bologna - 40138, Italy. E-mail: marco.pellegrini@hotmail.it

\section{Reference}

1. Singh M, Das S, Sangwan V. Comments on: Ocular surface status in patients with hemifacial spasm under long-lasting treatment with botulinum A toxin: A comparative fellow eye study. Indian J Ophthalmol 2020;68:264-5.

2. Pellegrini M, Schiavi C, Taroni L, Sebastiani S, Bernabei F, Roda M, et al. Ocular surface status in patients with hemifacial spasm under long- lasting treatment with botulinum A toxin: A comparative fellow eye study. Indian J Ophthalmol 2019;67:1405-9.

This is an open access journal, and articles are distributed under the terms of the Creative Commons Attribution-NonCommercial-ShareAlike 4.0 License, which allows others to remix, tweak, and build upon the work non-commercially, as long as appropriate credit is given and the new creations are licensed under the identical terms.

\begin{tabular}{|l|l|}
\hline \multicolumn{2}{|c|}{ Access this article online } \\
\hline Quick Response Code: & Website: \\
\cline { 1 - 2 } & www.ijo.in \\
\cline { 2 - 3 } & DOI: \\
\cline { 1 - 2 } & 10.4103/ijo.IJO_2025_19 \\
\hline
\end{tabular}

Cite this article as: Pellegrini M, Schiavi C, Taroni L, Sebastiani S, Bernabei F, Roda M, et al. Response to comments on: Ocular surface status in patients with hemifacial spasm under long-lasting treatment with botulinum A toxin: A comparative fellow eye study. Indian J Ophthalmol 2020;68:265.

(c) 2019 Indian Journal of Ophthalmology | Published by Wolters Kluwer - Medknow 